\title{
Sonographic Assessment of Benign Cystic Knee Lesions
}

Hoda Salah Darwish ${ }^{1^{*}}$ and Hanaa Ahmed Kamel ${ }^{2}$

${ }^{1}$ Department of Radio-Diagnosis, Faculty of Medicine, Suez Canal University, Egypt

${ }^{2}$ Department of Radio-Diagnosis, Faculty of Medicine, Tanta University, Egypt

*Corresponding author: Hoda Salah Darwish, Associate professor of Radio-Diagnosis, Faculty of Medicine, Suez Canal University, Egypt, E-mail: darwish.hoda@yahoo.com

Received date: September 22, 2018; Accepted date: October 08, 2018; Published date: October 15, 2018

Copyright: $@ 2018$ Darwish HS, et al. This is an open-access article distributed under the terms of the Creative Commons Attribution License, which permits unrestricted use, distribution, and reproduction in any medium, provided the original author and source are credited.

\section{Abstract}

Aim: Aim of our study is to assess the usefulness of ultrasound in assessment and diagnosing the knee bengin cysts and cysts-like lesions.

Material and Methods: Patients with MRI diagnosis of cystic lesions in and around the knee joint were selected. These patients underwent knee Musclo-skeletal ultra-sonography. A blind ultrasound study to the knee joint, with no information about MRI result, was done by a senior muscolskeletal ultrasound expert radiologist.

We included 73 patients presented to radiology department. We compare the results of US to MRI to assess the value of US in the diagnosis. Overall sensitivity, specificity, predictive values and diagnostic accuracy of ultrasound was evaluated keeping MRI as gold standard.

Results: The mean age of the patients was $16 \pm 45$ years, Females to males' ratio was 3:1. The collected data of imaging US features, with exact lesion location, the relationship with surrounding structures and clinical features such as sex, age and symptoms in addition to X-ray, and MRI findings allowing accurate diagnosis.

MRI study identified the 58/73 cystic lesions including 34 cases synovial cysts, 24 of them was popliteal cysts, 18 cases ganglion cysts, and 6 cases meniscal cysts. The ganglion cysts were as follows 3 was intra-articular, 13 extraarticular and 2 cases intraosseous cysts.

MRI identified 15/73 cyst-like" lesions as follows: 13 cases distended knee bursae, and only 2 miscellaneous cyst-like lesions (post traumatic hematoma). However US identified 50 cases cystic lesions including 31 cases synovial cysts, 15 cases ganglion cysts, and 4 cases meniscal cysts, and missing 3 cases synovial cysts, 3 cases ganglioin cyst and 2 case meniscal cysts. However it identified all 13 cases of distended knee bursae/bursitis, and the 2 miscellaneous cases of cyst-like lesion (post traumatic hematoma).

Of our all 73 patients with cystic/ cyst like lesion of knee joint diagnosed with MRI study, ultrasound detected 65 cases with a sensitivity of $89 \%$ and specificity of $100 \%$. Ultrasound had a diagnostic accuracy of $89 \%$.

Conclusion:Musclo-skleteal ultrasonography is low in cost, easily available, highly efficient and can be used in the assessment of the knee cysts and cysts-like lesions. It is sensitive and specific for diagnosis of cystic knee lesions; however, it lacks sensitivity for small cysts, intrarticular cysts and intraosseous cysts.

Keywords: Ultrasound; MRI; Knee cyst; Bursae; Recesses; Synovial cyst

\section{Introduction}

Cysts and cystic-appearing lesions in and around the knee are common and can create a diagnostic dilemma if one is not aware of the potential diagnoses and pitfalls [1]. Its clinical presentation depends on its location, size and relation with the surrounding structures [2]

The benign knee cysts were subdivided into categories as following: (1) synovial cysts, (2) ganglion cysts, and (3) meniscal cysts. The "cystlike" lesions were sub-classified into the following: (1) normal knee bursae (2) normal knee recesses and (3) miscellaneous cyst-like lesions [3].
Ultrasonography (USG) and magnetic resonance imaging (MRI) are the most useful imaging modalities in evaluating these cystic lesions. USG can distinguish cysts from solid lesions and assess the degree of vascularity within the lesion.

MRI can help delineate the location of the lesion in relation to anatomic structures and also determine if the lesion is cystic or solid when contrast is administered [1]. At magnetic resonance (MR) imaging, fluid-filled masses are usually hypointense relative to muscle with T1-weighted sequences and highly hyperintense with T2weighted or fluid-sensitive sequences due to the prolonged T2 relaxation time of water [4].

At US, simple cysts are typically well defined and anechoic, with deep acoustic enhancement. Other fluid-containing lesions such as 
abscesses and hematomas have a different appearance, with lowsignal-intensity internal echoes that may be homogeneous or heterogeneous. These lesions do not show deep enhancement, and they may have thick or irregular walls [5].

For accurate assessment of the "cystic structure", a radiologist should be able to identify typical MRI and ultrasound patterns that contribute in establishing the correct diagnosis and thus guiding specific therapy and avoiding unwarranted interventional procedures such as biopsy or arthroscopy.

To conclude, a variety of bursae, cysts, and cyst-like lesions exist about the knee joint, many of which have fairly characteristic locations and imaging features. Knowledge of these variants and lesions is essential for accurate diagnosis and to avoid mistaking them for aggressive soft tissue lesions, including sarcomas. The goal of our study is to evaluate MSK US imaging in diagnosis of benign cysts and cysts like lesion of the knee.

\section{Materials and Methods}

Human ethics committee approval for this study was obtained from the institutional review board of our Hospital. A total number of 63 patients whose MRI studies were done from June 2017 and April 2018 were retrieved using picture archiving and communication system and MRI were evaluated retrospectively.

Patients with MRI diagnosis of cystic lesions in and around the knee were selected. These patients underwent Musclo-skeletal ultrasonography of the knee joint. A blind ultrasound study to the knee joint, with no information about MRI result, was done by a senior muscolskeletal ultrasound expert radiologist.

All patients were examined by ultrasound using an ultrasound machine (HD II XE Ultrasound 2006, Philips medical system, Nederland B.V). A linear transducer (L12-5) was used for all the patients (B-Mode) to assess knee joint. Color Doppler Mode also used in all cases. Dynamic ultrasonography was done in some cases.

Patient history were included, the exact location of pain, aggravated factors, the duration of symptoms, modes of treatment, and the degree of functional limitation. We compare the results of US to MRI to assess the value of US in the diagnosis. Overall sensitivity, specificity, predictive values and diagnostic accuracy of ultrasound was evaluated keeping MRI as a reference gold standard.

\section{Ultrasound scanning technique of the knee}

According to EULAR Guidelines for musculoskeletal ultrasound in rheumatology (A), the ultrasound examination of the knee should be methodic and standardized. Transverse and longitudinal scans of knee structures are recommended, according to a multi-plannar scanning method and dynamic assessment including examination of anterior, medial, lateral and posterior knee.

Anterior knee: To evaluate, quadriceps tendon, suprapatellar and parapatellar joint recesses, femoral trochlea, the medial and lateral patellar retinacula, and patellar tendon. The anterior aspect of the knee is examined with the patient supine.

A knee flexion of approximately $20^{\circ}-30^{\circ}$ obtained by placing a small pillow beneath the popliteal space. Sagittal US images obtained in the midline while keeping the distal edge of the probe over the patella display the quadriceps tendon, then shifting the transducer on axial planes.
Medial knee: To evaluate medial collateral ligament and the tendons of the pes anserinus complex (sartorius, gracilis and semitendinosus. For examination of the medial knee, the patient is asked to rotate the leg externally while maintaining $20^{\circ}-30^{\circ}$ of knee flexion.

Place the transducer obliquely-oriented over the long-axis of the medial collateral ligament. Care should be taken to examine the entire length of this ligament. Dynamic scanning during valgus stress can improve the assessment of its integrity

Lateral knee: To evaluate iliotibial band, lateral collateral ligament For examination of the lateral knee, rotate the patient's leg internally while maintaining $20^{\circ}-30^{\circ}$ of knee flexion.

Posterior knee: To evaluate medial tendons (the sartorius, the gracilis, the semitendinosus tendon and semimembranosus tendons), semimembranosus-gastrocnemius bursa, popliteal neurovascular bundle and biceps femoris muscle tendon. For examination of the posterior knee, the patient is asked to lie prone with the knee extended.

MRI: All patients underwent MRI using MRI scanner (Optima TM MR $450 \mathrm{~W} 1.5$ Tesla). The patient is comfortably placed in the supine position to avoid pain, motion and compression of different muscle groups.

Coronal and axial spin-echo T1 weighted [repetition time (TR)/ echo time (TE), 650-800 ms/15-20 ms] and fast spin-echo T2 weighted (TR/TE, 3300-4550 ms/90-140 ms) sequences with fat suppression is performed. Alternative to the fat-suppressed T2 weighted sequence is a short-tau inversion-recovery (TR/TE, 3000$3665 \mathrm{~ms} / 15-35 \mathrm{~ms}$; inversion time, $150 \mathrm{~ms}$ ) sequence.

Images in a sagittal plane are occasionally acquired to match individual requirements, and intravenous administration of gadolinium-containing contrast material is advised as appropriate. All images were evaluated on workstation (SECTRA, SWEEDEN).

\section{Statistical analysis}

Data was evaluated by using statistical package for social sciences (SPSS) software version 10 for calculating percentages and frequencies. Overall sensitivity, specificity, predictive values and diagnostic accuracy of ultrasound was evaluated keeping MRI as gold standard.

\section{Results}

In our study, 73 patients were included; knee swelling was the main complain, associated pain was seen in 55 patients (75\%) and 45 patients showing knee osteoarthritis $(62 \%)$. The mean age of the patients was $16 \pm 45$ years, Females to males' ratio was $3: 1$.

The collected data of imaging US features, with exact lesion location, the relationship with surrounding structures and clinical features such as sex, age and symptoms in addition to X-ray, and MRI findings allowing accurate diagnosis.

MRI study identified the $58 / 73$ cystic lesions (88\%) and $15 / 73$ cystlike" lesions (22\%). The cystic lesions including; 34 cases synovial cysts, 24 of them was popliteal cysts (Figures 1A and 1B), 18 cases ganglion cysts, and 6 cases meniscal cysts.

The ganglion cysts were as follows 3 was intra-articular (Figure 2), 13 extra-articular (Figure 3 ) and 2 cases intraosseous cysts. 

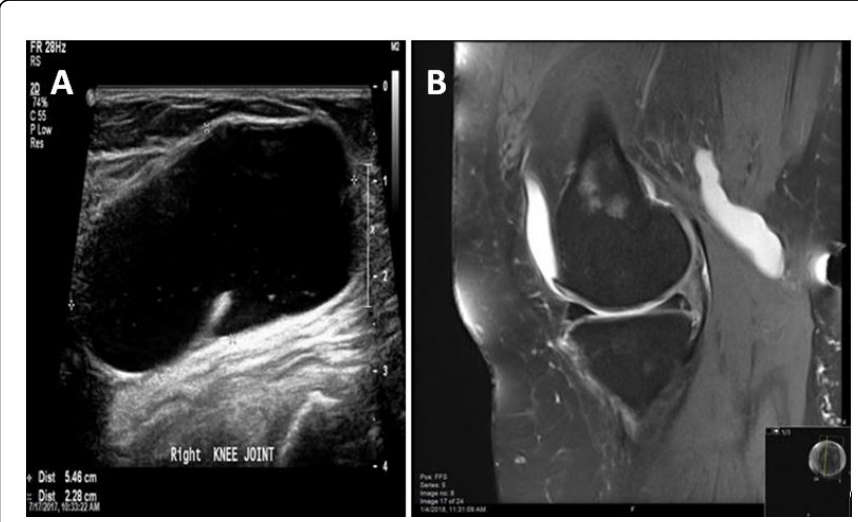

Figure 1: Popliteal cyst (backer's cyst), A 60-year-old female with posterior knee pain; (A) Gray scale USG image at the level of popliteal fossa demonstrates a large multilocular an echoic non vascular cystic lesion; (B) Sagittal p.d f/s through right knee demonstrates the hyperintense popliteal cyst fluid arising between semimembranosus (arrowhead) tendon and medial head of gastrocnemius.

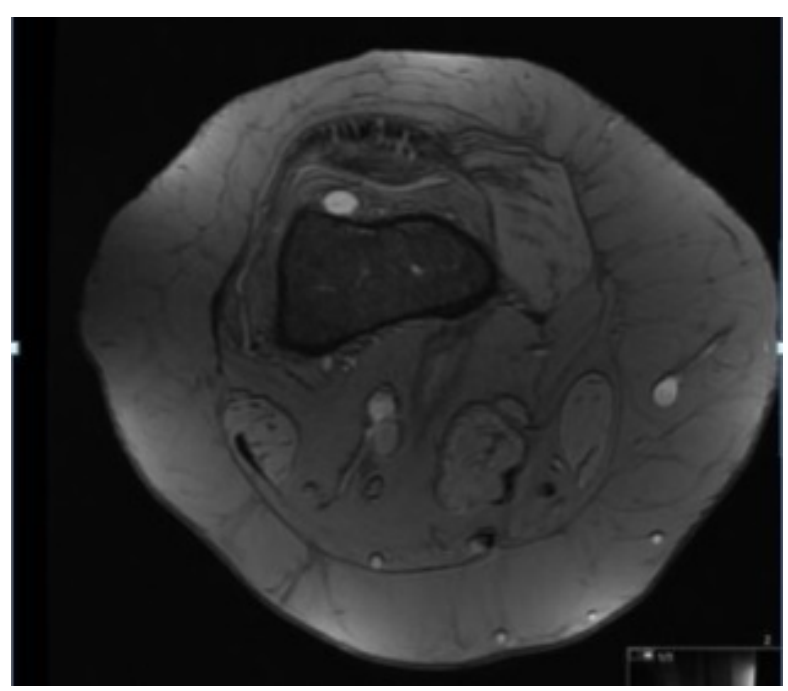

Figure 2: Axial T2 MERG image showing a small well defined patella-femoral intra-articular hyperintense cystic lesion suggesting intra-articular ganglioinc cyst.

MRI identified 15/73 cyst-like" lesions as follows: 13 cases distended knee bursae, and only 2 miscellaneous cyst-like lesions (post traumatic hematoma).

The distended knee burse was as follows 11 cases at anterior knee burse including the following: 2 cases prepatellar bursa, 2 cases superficial infrapatellar bursa (Figure 4), 5 cases deep infrapatellar bursa and 1 suprapatellar bursa. Another 2 cases distended medial gastrocnemius (MG) bursa was indentified with MRI study.

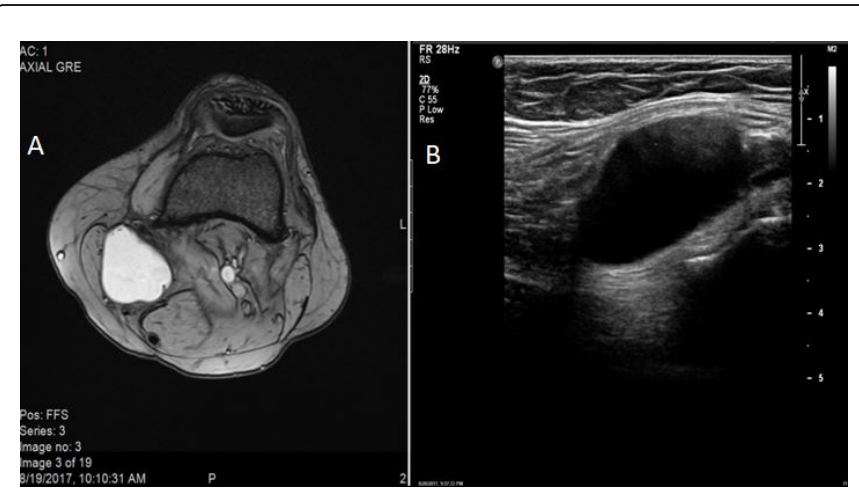

Figure 3: Extra-articular ganglionic cyst-38 years old male complaining of left knee pain; (A) Left knee US showing a well defined echofree cystic mass lesion at the posteromedial aspect of left knee related to the femoral medial condyle, not connected to the joint; (B) MRI axial GRE image; revealed hyperintense lesion suggesting extra-articular ganglionic cyst.

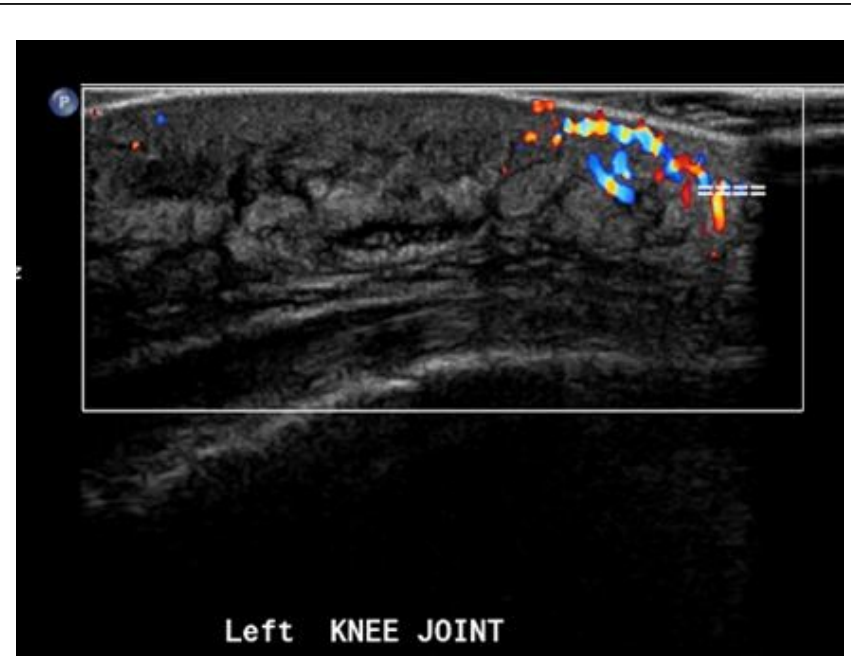

Figure 4: A 29-year-old male with pain and swelling of left knee. Left knee Color Doppler US L.A scan revealed features of prepatellar (superficial infrapatellar) bursitis with thickened hypoechoic bursa anterior to the patellar ligament, overlying subcutaneous edema associated with increased vascularity.

However US identified 50 cases cystic lesions including 31 cases synovial cysts, 24 of them were popliteal cysts, 15 cases ganglion cysts one of them was Hoffa fat pat ganglionic cyst (Figure 5), and 4 cases meniscal cysts, and missing 3 cases synovial cysts, 3 cases ganglioin cyst and 2 case meniscal cysts. 2 cases of missing 3 cases synovial cysts were polpliteal cysts.

The MS US of the knee identified all 13 cases of distended knee bursae/bursitis, and the 2 miscellaneous cases of cyst-like lesion (post traumatic hematoma) with no missing cases.

Of our all 73 patients with cystic/ cyst like lesion of knee joint diagnosed with MRI study, ultrasound detected 65 cases with a sensitivity of $89 \%$ and specificity of $100 \%$. 
Ultrasound had a diagnostic accuracy of $89 \%$. However out of 58 patients with true knee cystic lesion diagnosed with MRI study, 50 cases were diagnosed on ultrasound. The sensitivity and specificity were $86.2 \%$ and $100 \%$ respectively.

Eight patients with true cystic knee lesions were missed on ultrasound with a mean cyst size.

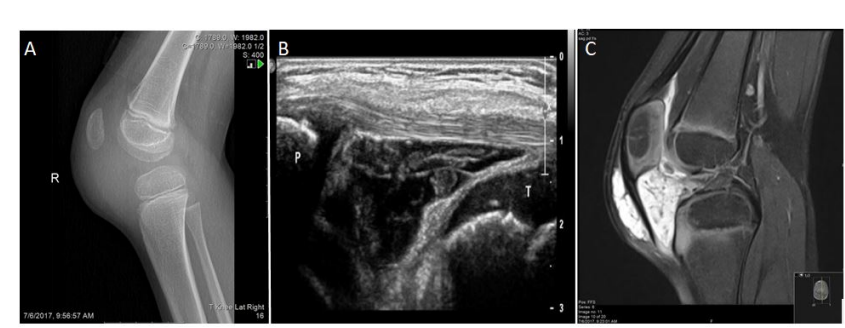

Figure 5: Hoffa fat pat ganglionic cyst-A 3-year-old male have right knee swelling; (A) Right knee X-ray lateral view showing marked soft tissue opacity; (B) Superficial Right knee ultrasound L.A scan; showing thickened hypoechoic deep infrapatellar bursa with overlying subcutaneous edema; (C) Right knee MRI sagittal pd, f/s; There is mutiloculated infrapatellar fat pad cystic lesion returning high signals with thin septations and extension antero-medially in the knee soft tissues encasing the patellar ligament.

\section{Discussion}

One of the more common imaging findings when evaluating the knee, whether by ultrasound or MRI, is the "cystic lesion, especially parameniscal cysts and popliteal cyst (Baker's cyst) [6]. Synovial cysts are a result of herniation of the synovial membrane through the joint capsule [7].

It is juxta-articular fluid collections and they are synovial lined, a feature which can normally be identified on post contrast images as peripheral enhancement in MRI study. Specific types of synovial cysts at the knee include the popliteal and tibiofibular cysts [8-10].

In our study, the most common cystic lesions was synovial cysts 31 cases, 24 of them was popliteal cysts, ultrasound missing 3 cases of synovial cysts 2 of them was polpliteal cysts, the 2 cases was smaller than $2 \mathrm{~cm}$ and one of patient was very obese make the diagnosis difficult.

Ganglion cyst is a benign cystic mass, have a fibrous lining with no synovial lining, surrounded by dense connective tissue and contain fluid or mucinous material, and it arise from the joint capsule, ligaments, tendon sheaths, bursae, or subchondral bone [2-7]. They are divided into: Intra-articular, extra-articular, intraosseous and (rare) periosteal [2].

We found that, the ultrasound missing the all 2 cases of intrarticular ganglion cysts and the all 2 cases of intraosseous ganglioinc cysts. This due to small sized of the intra-articular cysts and lack of sensitivity of US in diagnosis of intraosseous lesions.

We should mention that, in clinical practice, the terms ganglion cyst and synovial cyst are frequently used interchangeably due to the common peri-articular location of these lesions and the fact, in most cases, radiologic differentiation is not possible.
A normal bursa is not visualized on ultrasound, or is seen only as a thin hypoechoic space or sac in a typical anatomic location. When a bursa is distended, it appears as a hypoechoic structure with welldefined margins and contents of variable echogenicity [11]. These bursae can become distended from direct or repetitive trauma or secondary to local or systemic inflammatory changes. Bursitis can be further complicated by hemorrhage, rupture, synovial proliferation, chondromatosis, or infection [12].

Because of its excellent tissue contrast, multiplanar capability, and lack of ionizing radiation, MR imaging has become the modality of choice in the evaluation of soft-tissue masses. Its potential to help discriminate between solid and cystic lesions has been well demonstrated

However, ultrasonography has many diagnostic advantages, it should be applied in the primary clinic, dynamically and in real time, as shown in our study, it has the capability of demonstrating physiological movement, and is simpler and more cost effective than MRI.

In conclusion; Musclo-skleteal ultrasonography is low in cost, easily available, highly efficient and can be used in the assessment of the knee cysts and cysts-like lesions, from our study, it is sensitive and specific for diagnosis of cystic knee lesions; however, it lacks sensitivity for small cysts, intrarticular cysts and intraosseous cysts.

\section{References}

1. Telischak NA, Wu JS, Eisenberg RL (2014) Cysts and cystic-appearing lesions of the knee: A pictorial essay. Indian J Radiol Imaging 24: 182-191

2. Plotas, I Shaikh, K Latief (2015) Cystic and "cyst-like" lesions of the knee joint and around the knee: A pictorial essay in MR imaging. Poster No: C-2338

3. Perdikakis E, Skiadas V (2013) MRI characteristics of cysts and "cyst-like" lesions in and around the knee: what the radiologist needs to know. Insights Imaging 4: 257-272

4. Wu JS, Hochman MG (2009) Soft-tissue tumors and tumorlike lesions: A systematic imaging approach. Radiology 253: 297-316.

5. Johnstone AJ, Beggs I (1994) Ultrasound imaging of soft-tissue masses in the extremities. J Bone Joint Surg Br 76: 688-689.

6. Bermejo A, De Bustamante TD, Martinez A, Carrera R Zabía E, Manjón P MR (2013) Imaging in the evaluation of cystic-appearing soft-tissue masses of the extremities. Radiographics 33: 833-55.

7. Janzen DL, Peterfy CG, Forbes JR, Tirman PF, Genant HK (1994) Cystic lesions around the knee joint: MR imaging findings. AJR Am J Roentgenol 163: 155-161.

8. Steiner E, Steinbach LS, Schnarkowski P (1996) Ganglia and cysts around joints. Radiol Clin North Am 34: 400-410.

9. Miller TT, Starson RB, Koenigsberg T (1996) MR imaging of Baker cysts: Association with internal derangement, effusion and degenerative arthropathy. Radiology 201: 247-250.

10. Torreggiani WC, Al-Ismail K, Munk PL (2002) The imaging spectrum of Baker's (popliteal) cysts. Clin Radiol 57: 681-691.

11. Backhaus M, Burmester GR, Gerber T (2001) EULAR Guidelines for musculoskeletal ultrasound in rheumatology. Ann Rheum Dis 60: 641-649

12. Wang SC, Chhem RK, Cardinal E, Cho KH (1999) Joint sonography. Radiol Clin North Am 37: 653-668 